\title{
The Application for Harmonic Superposition in Wind Farm Using the Methodology of Monte-Carlo
}

\author{
Jiayi Wang ${ }^{1}$, Yanchi Zhang ${ }^{1, *}$, Hongkun Yang ${ }^{2}$, Xiangping $\mathrm{Xu}^{1}$ and Yi Zha ${ }^{1}$ \\ ${ }^{1}$ School of Electrical Engineering, Shanghai Dianji University, Shanghai 201306, China \\ ${ }^{2}$ Shanghai Electric Wind Power Equipment Co., Ltd, Shanghai 200241, China \\ ${ }^{*}$ Corresponding author
}

\begin{abstract}
The harmonic aggregation in wind power generation is widely concerned in engineering practice. In this paper, large volumes of voltage and current data in wind farm was measured and analyzed for exploring the distribution rules of harmonic current amplitude and phase, based on which, the probabilistic statistical model of harmonic current in wind farm was set up. And the superposition of harmonic in wind farm was predicted and evaluated with Monte-Carlo method. Simulation shows that the calculation of harmonic superposition with Monte-Carlo method has strong reliability and maneuverability. This method can be used to calculate the mean value of the superposition of harmonic and predict the harmonic aggregation at extreme situations.
\end{abstract}

Keywords-wind farm; harmonic superposition; harmonic current phase; probability distribution; monte-carlo method

\section{INTRODUCTION}

The renewable energy industries have been greatly developed in recent years due to the global energy crisis. Among them, wind power has become one of the dominate renewable energy resources because of its low cost and high capacity. According to the latest report of world wind energy (WWEA)[1], the global installed capacity has reached 370GW since the end of 2014. Meanwhile, the installed scale of wind farms is gradually increasing and the rapid expansion of the nonlinear load, causing the world's attention on power quality issues.

Increasing installed capacity of wind power plant has contributed to the variation of harmonic current distortion rate at the side of main transformer of wind farm. According to the testing reports which manufacturer released, in ideal working condition of balance grid voltage, total current distortion rate will reach 3\% with rated power of wind turbines, but reach more than $10 \%$ during actual operation. Otherwise , it is hard to meet the relevant standard of grid integration[2-4].The main reasons of this phenomenon are aroused from the discreteness of harmonic current phase angles produced by each unit in power plant, which makes the situation of same phase angle happened and leads to increasing current distortion rate, poor power quality, incidental resonance with grid, overcurrent and overvoltage. At the same time, further harmonic response will happened in resonance and it might damage the electrical equipment more seriously. Under these situations, the improvement of power quality has become the key point in wind power research.
Previous research work on wind power harmonic current problem mainly concentrated on converter control strategies in the single-unit system[5-6]. But in wind farm, harmonic current has recently made impact on power grid. So problems related to harmonic resonance have been analyzed through different analysis methods[7].

Nowadays, the growing use of power electronics systems in the grid verifies the importance of the research of harmonic problems[8].The deterministic and stochastic characterization of harmonic currents amplitude and phase angle is analyzed through an 18MW wind farm[9].As we all know, few research on the harmonic model of wind farm and the analysis of harmonic current have been performed, whereas, the research on harmonic current and traction load system has developed a lot [10-13].

In order to understand the harmonic distribution of wind farms and establish wind farms harmonic probability model precisely, this paper develops the study of harmonic model on the basis of the large volumes of measured data in wind farms, using the stochastic process theory and numerical analysis theory. This paper will simulate the wind farm harmonic phase superposition situation with Monte-Carlo method, and analyze the factors affecting the grid at the point of common coupling (PCC). To suppress the harmonic current of wind farm connected to power grid, providing a reliable assessment can reduce the loss of human and financial resources in the post-equipment renovation project.

\section{Generating Principles Of The Dominiant HARMONIC COMPONENT}

The main harmonic source of doubly-fed Induction Generator (DFIG) is the converter, the control strategies through the closed loop makes the output waveform and the target positive sequence identical, there are mainly three types of closed-loop control, hysteresis control, sinusoidal pulse width modulation (SPWM) and space voltage vector pulse width modulation (SVPWM).In the three ways of closed-loop control, the $6 \mathrm{~N}+1$ harmonic generation is inevitable due to the influence of the dead time of the IGBT switch. Considering the situation of stator and rotor working together at the same time in DFIG, the three-phase AC voltage is set up as follows: 


$$
e_{i}=\sum_{m=1}^{\infty} \sum_{s} E_{m s} \cos \left(\omega_{m} t+\theta_{i}\right)
$$

where $i$ is $a, b, c$, three phase and $s$ is $p, n, 0$ which represent positive sequence; negative sequence and zero sequence respectively.

During rectification and DC voltage represented as follows:

$$
\begin{aligned}
u_{d c}= & \sum_{m=1}^{\infty} \sum_{n=1}^{\infty} \sum_{s} 0.5 E_{m s} A_{n u}((1+2 \cos (2 \pi(n+s) / 3) \times \\
& \sin \left(\left(\omega_{m}+n \omega_{1}\right) t+\theta_{a}\right)+(1+2 \cos (2 \pi(n-s) / 3) \\
& \left.\times \sin \left(\left(\omega_{m}-n \omega_{1}\right) t+\theta_{a}\right)\right)
\end{aligned}
$$

where $A_{n u}$ is the corresponding coefficient of voltage switching function for converter control and $\omega_{1}$ is the modulation wave frequency.

At present, space vector control as the most common method is used in convertor control. It has higher utilization ratio for DC voltage, easier performance in digitization and the relative lower harmonic than other methods. At the same time, considering the process of grid integration which power transits from DC side to grid-side convertor, the space vector control is used by similar control method of phase control by six IGBTs of three phase inverter bridge. The analysis method of phase controlled inverter keeps on being used to express. The switching function of three-phase 6 pulse converter is represented as follows:

$$
\begin{aligned}
& S_{i a}=k_{1} \cos \left(\omega_{2} t\right) \\
& -\sum_{j=1}^{\infty} k_{6 j-1} \cos \left((6 j-1) \omega_{2} t\right)-k_{6 j+1} \cos \left((6 j+1) \omega_{2} t\right)
\end{aligned}
$$

where $k_{i}$ Indicates the coefficient of each component and $\omega_{2}$ represents modulation frequency of the grid-side converter.

DC current corresponding to (3) is expressed as:

$$
\begin{aligned}
& i_{d c}=I_{d c}+\sum_{k=0}^{\infty} I_{(6 k)} \sin \left(6 k \omega_{1} t+\theta_{k p}\right)+\sum_{k=0}^{\infty}\left(I_{(6 k+1)}\right. \\
& \left.\sin \left((6 k+2) \omega_{1} t+\theta_{k n}\right)+I_{(6 k-1)} \sin \left((-6 k+2) \omega_{1} t+\theta_{k n}\right)\right)+ \\
& \sum_{m=1}^{\infty} \sum_{k=1}^{\infty}\left(I_{(6 k+1) m} \sin \left((-6 k+1) \omega_{1} t+\omega_{m} t+\theta_{k m}\right)+I_{(6 k-1) m}\right. \\
& \left.\sin \left((6 k-1) \omega_{1} t+\omega_{m} t+\theta_{k m}\right)\right)
\end{aligned}
$$

In (4), the results is divided into four parts which are separately the corresponding effect of DC component, the influence of fundamental voltage positive sequence, negative sequence and each harmonic component in AC side of rectifier on the current of DC side.
The output voltage of the grid-side converter is $(\mathrm{B}, \mathrm{C}$ are similar):

$$
i_{a}=i_{d c} s_{i a}
$$

Put(3),(4)into(5),we can get dominant harmonic components.

\section{MeAsurement And AnAlysis Of Harmonic DatA}

\section{A. Method of Measurement}

The measurement method on power quality of wind power mentioned in the designing fourth version of IEC61400-21 will be used in this paper. Its basic calculation method is to analyze the $200 \mathrm{~ms}$ data within the 10 cycles by FFT when the sampling frequency ranging from $10 \mathrm{kHz}$ to $20 \mathrm{kHz}$. And then the amplitude and phase of the harmonics are obtained. FFT as a mature algorithm, the basic principle is not introduced in detail.

In this paper, the basic calculation process of the collected 2048 data points in each section was analyzed by FFT.

$$
X(\mathrm{k})=\operatorname{FFT}[\mathrm{x}(\mathrm{n})] \mathrm{n}=0,1,2 \cdots, 2047
$$

where $x(n)$ is one of the sampling values of the three phase voltage and current A B C.

The plural result $X(\mathrm{k})$ by FFT is acquired. Through the results, we can calculate the amplitude and phase angle values of harmonic voltage and current. During MATLAB programming, the resolution frequency of $5 \mathrm{~Hz}$ is chosen. ABS is calculation of amplitude and Angle is function of phase angle. The process of calculation is as follows:

Voltage DC component:

$$
\mathrm{U}_{\mathrm{dc}}=X(0) / 2048
$$

Fundamental voltage amplitude:

$$
\begin{aligned}
& \mathrm{U}_{1}=\mathrm{ABS}[X(10)] / 1024 \\
& =\sqrt{\operatorname{Re}^{2}[X(10)]+\operatorname{Im}^{2}[X(10)]} / 1024
\end{aligned}
$$

Fundamental voltage phase:

$$
\angle \mathrm{U}_{1}=\operatorname{Angle}[X(10)]=\operatorname{arctg} \frac{\operatorname{Im}[\mathrm{X}(10)]}{\operatorname{Re}[\mathrm{X}(10)]}=\alpha
$$

$\mathrm{N}^{\text {th }}$ harmonic voltage amplitude:

$$
\mathrm{U}_{n}=\mathrm{ABS}[X(10 n)] / 1024
$$


$\mathrm{N}^{\text {th }}$ voltage harmonic phase:

$$
\angle \mathrm{U}_{n}=\operatorname{Angle}[X(10 n)]-\mathrm{n} \alpha
$$

$\mathrm{N}^{\text {th }}$ harmonic current phase:

$$
\angle I_{n}=\text { Angle }\left[X_{i}(10 n)\right]-\mathrm{n} \alpha
$$

In the actual data analysis, the accuracy of the phase value of the fundamental voltage should be in a high standard because there is no uniform time standard based actual sampling data of each wind turbine units when the sampling work happened. For acquiring the phase relationship of different frequency harmonics of output current in wind turbine, calculation must be based on a common time starting point. At this time, harmonic currents of each units in wind farm gather at the point of common coupling (PCC)-the main transfers side. During the progress of calculation, each wind turbine should be the same type, the transformer connected should be same as well. Transmission distance of each unit in the wind farm is slightly different, so the total impedance has little difference. Then the fundamental phase is the reference value when calculating the phase value of each harmonic. Besides, the accuracy of calculation for each harmonic would be highly influenced by very small deviation of fundamental phase.

\section{B. Analysis of Harmonic Based on One Wind Turbine}

The gathered data in this paper is from wind farm including 50 sets of Doubled-Fed wind power generator. The framework of a DFIG is shown in FIGURE I. Cable lines carry the output current of each wind turbines to the medium voltage collector with $35 / 0.69 \mathrm{kV}$ transformers. Through transmission line and $110 / 35 \mathrm{kV}$ transformer, currents are fed into the grid.

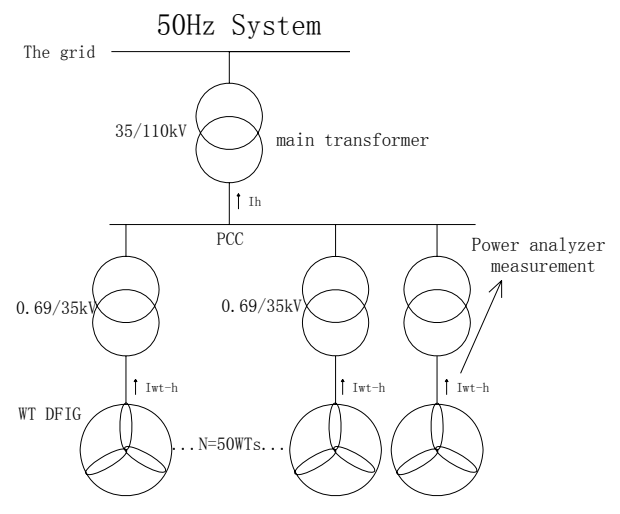

FIGURE I. WIND FARM TOPOLOGY.

One 2MW Doubled-Fed wind power generator is randomly selected for real-time data acquisition. 103 groups of data during 14 days, which is three phase voltage and current output waveform of the wind turbine in wind speed between $3 \mathrm{~m} / \mathrm{s}$ to $10 \mathrm{~m} / \mathrm{s}$,are obtained. The time span of each group is 10 s and the probability density functions of wind speed and power are separately shown in FIGURE II.
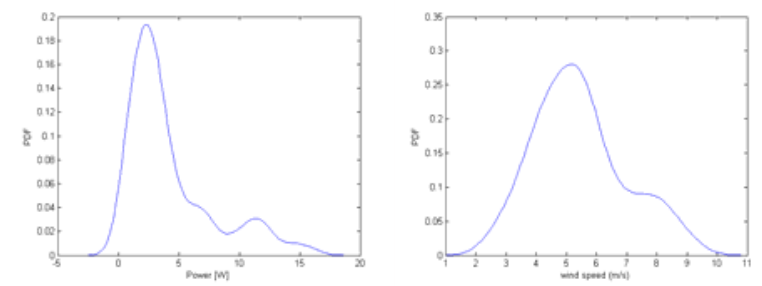

FIGURE II. PROBABILITY DENSITY FUNCTION OF WIND SPEED AND POWER OUTPUT DATA.

The data segment of 200ms intercepted from collected data by DEWE soft is analyzed and calculated. Its sampling frequency is $4 \mathrm{kHz}$ and the frequency resolution is $5 \mathrm{~Hz}$. Through statistical calculation, the probability density function (PDF) of $6 n \pm 1$ th harmonics are shown in FIGURE III, which distribution characteristics is approximately considered as the normal distribution. As sample size increasing, the statistics are closer to the normal distribution. So probabilistic models of harmonic amplitude in wind farm have been effectively built.
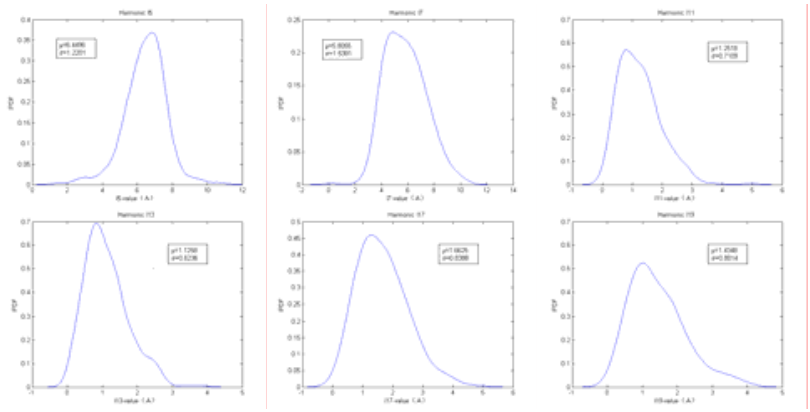

FIGURE III. STATISTICAL ANALYSIS OF HARMONIC CURRENT DURING THE WIND SPEED RANGED FROM 2.8M/S TO 9.2M/S IN 2MW DFIG.

\section{Harmonic Distribution Characteristic of Wind Farm}

The relations between $6 n \pm 1$ th harmonic current amplitude and phase produced by wind turbines in wind farm are shown in FIGURE.IV. Obviously, the phases of the fifth and seventh harmonic current are relatively stable and have less fluctuation. And yet, $11^{\text {th }}, 13^{\text {th }} 17^{\text {th }}$ and $19^{\text {th }}$ harmonic current amplitudes change little, but the phase have large fluctuations. It results from the features of more than $11^{\text {th }}$ harmonic which are high frequency and short period. A slight error caused by the measurement of the fundamental phase angle can make phase angle generate a lot of volatility. 

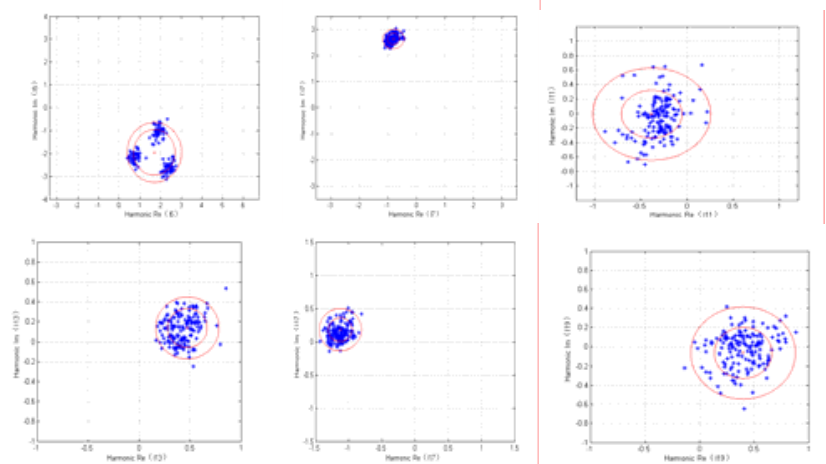

FIGURE IV. DISTRIBUTION OF HARMONIC CURRENT IN WIND FARM.

\section{Statistical Model OF HaRmonic In Wind FARM}

Considering situation of the output current in box transformer substation during operation of wind turbine, when harmonic currents from different wind turbines go through transmission lines and gather together, the output harmonic current of main transformer will be thought to be approximately the summation of them. $\mathrm{N}^{\text {th }}$ harmonic current can be expressed as:

$$
I_{n}=\sum_{j=1}^{N} X_{j n}+j \sum_{j=1}^{N} Y_{j n}=X_{t}+j Y_{t}
$$

The distribution of the harmonic current in the wind farm is mainly dependent on the statistical characteristics of the harmonic current of the single WT (PDF of amplitude and phase). For $50 * 2 \mathrm{MW}$ DFIGs in wind farm, their dominant harmonic components (such as $5^{\text {th }}, 7^{\text {th }}, 11^{\text {th }}, 13^{\text {th }}, 17^{\text {th }}$ and $19^{\text {th }}$ harmonics) are independent respectively. According to the central limit theorem, $X_{j}$ and $Y_{j}$ can be obtained and approximately obey the normal distribution. $X_{j n}$ and $Y_{j n}$ have own physical characteristics, so their expectation and variance can be almost a certainty. As long as value of $\mathrm{N}$ is large enough, we can deduce and attain that $X_{j}$ and $Y_{j}$ can obey normal distribution based on law of large numbers and central limit theorem. Through statistics, the dominant harmonic component in wind farm are shown in FIGURE V.
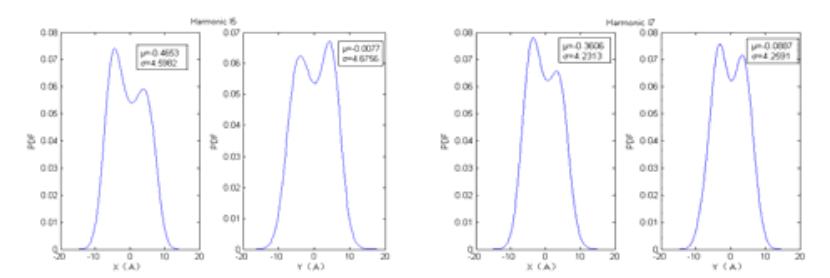
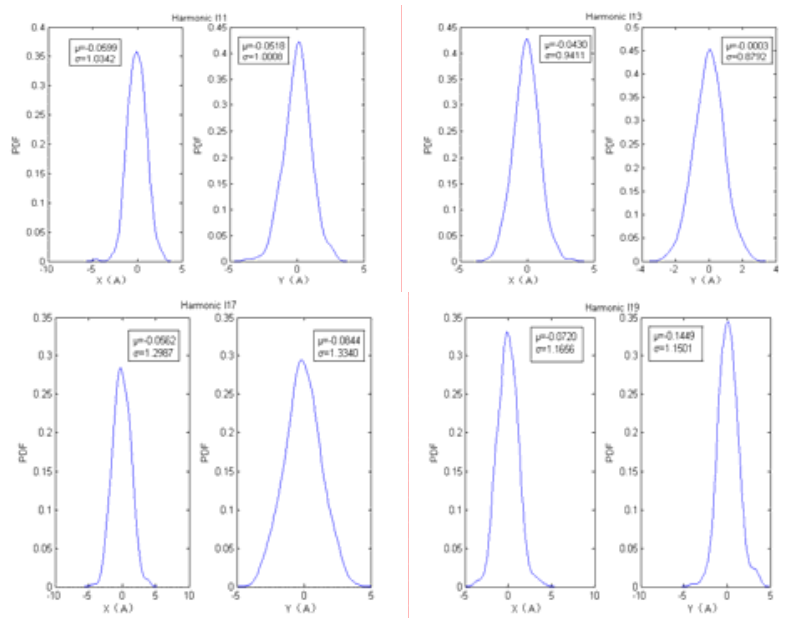

FIGURE V. STATISTICAL ANALYSIS OF THE HARMONIC CURRENT $X-Y$ PROJECTION.

The joint distribution of $X_{i}$ and $Y_{j}$ is determined by the following 5 parameters: $\mu_{X_{t}}, \mu_{Y_{t}} \sigma_{X_{t}}, \sigma_{Y_{t}}, \rho_{t}$. Among them, $\mu_{X_{t}}$ and $\mu_{Y_{t}}$ are the mathematical expectation of $X_{t}$ and $Y_{t} ; \sigma_{X_{t}}$ and $\sigma_{Y_{t}}$ are the standard variance of $X_{t}$ and $Y_{t} ; \rho_{t}$ is the correlation coefficient of $X_{t}$ and $Y_{t}[9,14]$. Joint density function is expressed as:

$$
\begin{aligned}
& f_{x y}\left(X_{t}, Y_{t}\right)=\frac{1}{2 \pi \sigma_{X_{t}} \sigma_{Y_{t}}} \exp \left\{\frac { - 1 } { 2 ( 1 - \rho _ { t } ^ { 2 } ) } \left[\frac{\left(X_{t}-\mu_{X_{t}}\right)^{2}}{\sigma_{X_{t}}^{2}}-\right.\right. \\
& \left.\left.\frac{2 \rho_{t}\left(X_{t}-\mu_{X_{t}}\right)\left(Y_{t}-\mu_{Y_{t}}\right)}{\sigma_{X_{t}} \sigma_{Y_{t}}}+\frac{\left(Y_{t}-\mu_{Y_{t}}\right)^{2}}{\sigma_{Y_{t}}^{2}}\right]\right\}
\end{aligned}
$$

The expectation and variance of the algorithm are no longer duplicated, and the joint distribution of the 5 parameters can be obtained by the following:

$$
\begin{aligned}
& \mu_{X_{t}}=\sum_{j=1}^{N} \mu_{X_{j}} ; \mu_{Y_{t}}=\sum_{j=1}^{N} \mu_{Y_{j}} ; \sigma_{X_{t}}=\left(\sum_{j=1}^{N} \sigma_{X_{j}}^{2}\right)^{1 / 2} ; \sigma_{Y_{t}}=\left(\sum_{j=1}^{N} \sigma_{Y_{j}}^{2}\right)^{1 / 2} ; \\
& \rho_{t}=\operatorname{cov}\left(X_{t}, Y_{t}\right) /\left(\sigma_{X_{t}}, \sigma_{Y_{t}}\right)=\sum_{j=1}^{N}\left(\rho_{t j} \sigma_{X_{j}} \sigma_{Y_{j}}\right) /\left(\sigma_{X_{t}} \sigma_{Y_{t}}\right)
\end{aligned}
$$

Thus, the probability density function of the vector $I_{h}$ will be acquired. $X_{t}=r \cos \theta$ and $Y_{t}=r \sin \theta$ will be plugged into (14) and get the joint density function:

$$
f_{r}(r)=\int_{0}^{2 \pi} f(r \cos \theta, r \sin \theta) r d \theta
$$

By calculation, when the harmonic current amplitude and phase value produced by the power generating units are satisfied with the assumption, that is the real and imaginary parts of the harmonic currents can satisfy the condition of the 
independence for each other and the same distribution, the distribution function of harmonic current and the statistical characteristics of the harmonic current amplitude can be obtained.

\section{Simulating Based On Monte-Carlo Method}

The basic principle of Monte Carlo method is based on Bernoulli's law of large numbers. If the probability $P\left\{X_{i}\right\}$ of random events $X_{i}$, in the independent sampling $\mathrm{N}$, the frequency of the incident is followed as $\mathrm{m} / \mathrm{N}(\mathrm{m}$ is the number of events $X_{i}$ in n trials)[15,16]. For any small positive $\varepsilon>0$ given, we get:

$$
\lim _{N \rightarrow \infty} P\left\{\left|\frac{m}{N}-P\left\{\mathrm{X}_{i}\right\}\right|<\varepsilon\right\}=1
$$

That is to say, when the number $N$ of independent experiments is large enough, the frequency of the $m / N$ is convergent to $P\left\{X_{i}\right\}$. This ensures the probabilistic convergence of the simulation method.

For the phase distribution of the wind farm and the harmonic data of the single unit under different wind speeds acquired, the model of the dominant harmonic component in the wind farm is established. The process diagram of simulating specifically with Monte-Carlo method is shown in FIGURE VI. by this method, the harmonic current summation at the point of common coupling can be obtained, and the power quality can be effectively evaluated. The extreme situation of the harmonic current in the wind farm can be analyzed and judged, meanwhile the mean values are acquired.

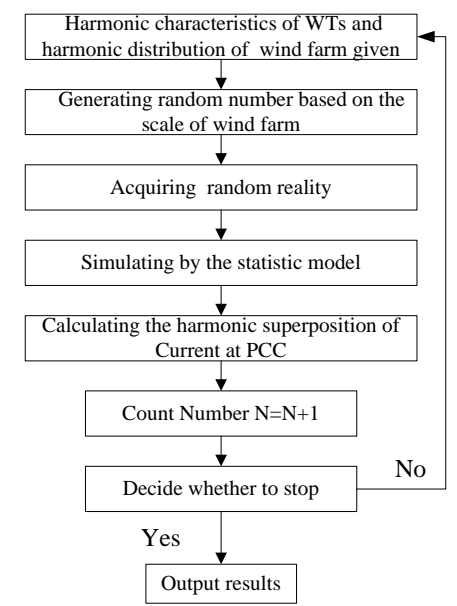

FIGURE VI. THE PROCESS DIAGRAM OF SIMULATION WITH MONTE-CARLO METHOD.

Utilizing Monte-Carlo method can obtain the evaluation values of the harmonic current at the point of common coupling. Results of simulation are shown as FIGURE VII After the harmonic currents go through the $0.69 / 35 \mathrm{kV}$ box transformer substations, the mean value of the $5^{\text {th }}$ and $7^{\text {th }}$ harmonic currents at the low-voltage side of the main transformer is 8 to $9 \mathrm{~A}$, while $11^{\text {th }}, 13^{\text {th }}, 17^{\text {th }}$ and $19^{\text {th }}$ harmonic currents are superimposed around the average value of $2 \mathrm{~A}$. It is not hard to see that the dominant harmonic source of the wind power system is $6 n \pm 1$ harmonics caused by the dead time in the converter control. In addition, we have evaluated the worst condition of the harmonic current in power grid, that amplitude peaks of $5^{\text {th }}$ and $7^{\text {th }}$ harmonics is over $16 \mathrm{~A}$ and $11^{\text {th }}$, $13^{\text {th }}, 17^{\text {th }}$ and $19^{\text {th }}$ harmonic current peaks reach $5 \mathrm{~A}$. It fully shows the importance of study on the phase of the network harmonic current. In order to evaluate the variation of the harmonic current in wind farm, it's important to establish the statistical model by actual measurement data. Meanwhile, according to $P=n / N$, combined with the actual requirements of the harmonic current, its probability exceeding the limit value will be acquired, and it's convenient to assess and predict power quality problems in the wind farm. The superposition result of the harmonics is also similar to the normal distribution, which provides a strong basis for establishing harmonic model of the wind farm.
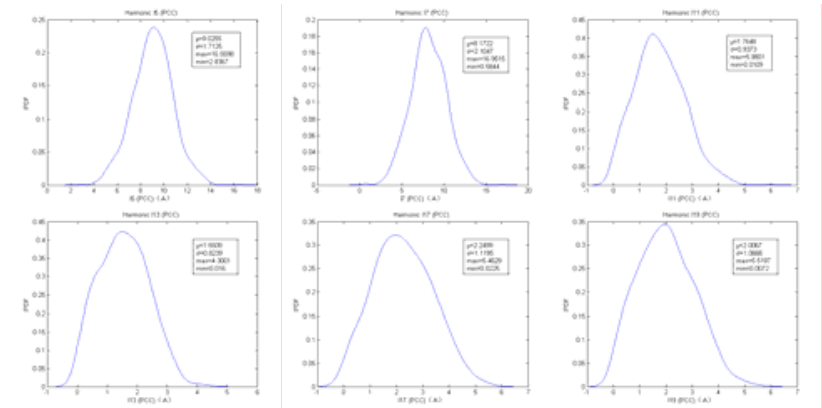

FIGURE VII. PROBABILITY DENSITY PLOT OF SIMULATION WITH MONTE-CARLO METHOD.

\section{CONCLUSION}

A systematic method has presented in this paper for analyzing and evaluating the harmonic current in wind farm. The key contributions of the paper are:(1) utilize measured data to analyze the amplitude and phase angle of the dominant harmonic current components and acquiring its PDF, (2)set uo the statistic model of harmonic current of wind farm suitable for calculation of harmonic current (3) predict and evaluat the harmonic current values at the point of common coupling based on the Monte-Carlo method.

This comprehensive method shown in this paper is benefit to forecast and evaluate power quality at PCC, which can be used to avoid the impact of harmonic and reduce the investment of regular maintenance for the company. The evaluation method not only creates a strong foundation to reduce harmonic superposition by phase interleaving, but also provides a practical reference for adjusting the wind power equipment and the optimization of control strategy.

\section{ACKNOWLEDGMENT}

This work has been funded by the National Natural Science Foundation of China (51277119). 
This work is supported by Shanghai education commission, science and technology innovation fund project(10Y217).

\section{REFERENCES}

[1] WWE Association, "WWEA half year report 2014," (2014)

[2] IEC 61400-21 Wind turbine generator systems, Part 21: Measurement and assessment of power quality characteristics of grid connected wind turbines.

[3] IEC 61000-3-6 Electromagnetic Compatibility (EMC) Part 3: LimitsSection 6: Assessment of emission limits for distorting loads inMW and HV power systems-Basic EMC Publication

[4] IEC 61000-4-7 Electromagnetic compatibility (EMC) Part 4-7: Testing and measurement techniques-General guide on harmonic and interharmonic measurements and instrumentation, for power supply systems and equipment connected thereto.

[5] Chen Y, Tong Y, Jin X. A novel algorithm of SVPWM harmonic analysis[C]//Industrial Electronics and Applications, 2007. ICIEA 2007. 2nd IEEE Conference on. IEEE, 2007: 1752-1755.

[6] Hughes F M, Anaya-Lara O, Jenkins N, et al. Control of DFIG-based wind generation for power network support[J]. Power Systems, IEEE Transactions on, 2005, 20(4): 1958-1966.

[7] Hasan K N M, Rauma K, Rodriguez P, et al. An overview of harmonic analysis and resonances of large wind power plant[C]/IECON 201137th Annual Conference on IEEE Industrial Electronics Society. IEEE, 2011: 2467-2474.

[8] Yu N, Nian H, Quan Y. A novel DC grid connected DFIG system with active power filter based on predictive current control[C]//Electrical Machines and Systems (ICEMS), 2011 International Conference on. IEEE, 2011: 1-5.

[9] Sainz L, Mesas J J, Teodorescu R, et al. Deterministic and stochastic study of wind farm harmonic currents[J]. Energy Conversion, IEEE Transactions on, 2010, 25(4): 1071-1080.

[10] Papathanassiou S, Papadopoulos M P. Harmonic analysis in a power system with wind generation[J]. Power Delivery, IEEE Transactions on, 2006, 21(4).

[11] Vasile R, Galve F, Zambrini R. Spectral origin of non-Markovian opensystem dynamics: A finite harmonic model without approximations[J].Physical Review A, 2014, 89(2): 022109.

[12] Xie S, Li Q, Zhao L. Study on harmonic distribution characteristic and probability model of the traction load of electrified railway[J]. PROCEEDINGS-CHINESE SOCIETY OF ELECTRICAL ENGINEERING, 2005, 25(16): 79.

[13] Cella U, Tran T, Nussey P, et al. Modelling of traction power system for evaluation of harmonic distortion in relation to route capacity[C]//CORE 2014, Rail transport for a vital economy, conference on railway engineering, Adelaide, South Australia, 5-7 May 2014. 2014.

[14] Baghzouz Y, Tan O T. Probabilistic modeling of power system harmonics[J]. Industry Applications, IEEE Transactions on, 1987 (1): 173-180.

[15] Kazibwe W E, Ortmeyer T H, Hammam M. Summation of probabilistic harmonic vectors [power systems][J]. Power Delivery, IEEE Transactions on, 1989, 4(1): 621-628.

[16] El-Khattam W, Hegazy Y G, Salama M M A. Investigating distributed generation systems performance using Monte Carlo simulation[J]. Power systems, IEEE transactions on, 2006, 21(2): 524-532. 